Research Article

\title{
Block Hybrid $k$-Step Backward Differentiation Formulas for Large Stiff Systems
}

\author{
S. N. Jator ${ }^{1}$ and E. Agyingi ${ }^{2}$ \\ ${ }^{1}$ Department of Mathematics and Statistics, Austin Peay State University, Clarksville, TN 37044, USA \\ ${ }^{2}$ School of Mathematical Sciences, Rochester Institute of Technology, Rochester, NY 14623-5603, USA \\ Correspondence should be addressed to S. N. Jator; jators@apsu.edu
}

Received 16 June 2014; Revised 29 September 2014; Accepted 30 September 2014; Published 20 October 2014

Academic Editor: Zhijie Xu

Copyright (c) 2014 S. N. Jator and E. Agyingi. This is an open access article distributed under the Creative Commons Attribution License, which permits unrestricted use, distribution, and reproduction in any medium, provided the original work is properly cited.

This paper presents a generalized high order block hybrid $k$-step backward differentiation formula (HBDF) for solving stiff systems, including large systems resulting from the semidiscretization parabolic partial differential equations (PDEs). A block scheme in which two off-grid points are specified by the zeros of the second degree Chebyshev polynomial of the first kind is examined for convergence, $L$ and $A$ stabilities. Numerical simulations that illustrate the accuracy of a Chebyshev based method are given for selected stiff systems and partial differential equations.

\section{Introduction}

We consider the first order differential equation

$$
y^{\prime}(t)=f(t, y(t)), \quad y\left(t_{0}\right)=y_{0}, \quad t \in\left[t_{0}, t_{N}\right],
$$

where $N \in \mathbb{Z}^{+}, f: \mathfrak{R} \times \mathfrak{R}^{m} \rightarrow \mathfrak{R}^{m}, y, y_{0} \in \mathfrak{R}^{m}, f$ satisfies a Lipschitz condition, and the eigenvalues of the Jacobian $(\partial f / \partial y)$ have negative real parts (see [1]). It is well known that the system (1) is better handled by methods with larger stability intervals. In particular, $A$-stable methods are of great importance. However, for very large systems arising from the semidiscretization of parabolic PDEs, $A$-stable methods converge very slowly to the exact solution. Hence, we seek methods which are at least $L_{0}$-stable for efficiently solving (1) when the system is very large (see Cash [2]).

The development of continuous methods has been the subject of growing interest due to the fact that continuous methods enjoy certain advantages, such as the potential for them to provide defect control (see Enright [3]) as well as having the ability to generate additional methods, which are combined and applied in block form (see Onumanyi et al. $[4,5]$, Akinfenwa et al. [6], and Jator [7]).

The majority of block methods which are due to Shampine and Watts [8], Chartier [9], Rosser [10], and Chu and Hamilton [11] are generally implemented in the predictor-corrector mode. In this paper, we adopt a different approach where the solution is simultaneously provided in each block (see Jator et al. [12], Jator [7, 13]) without the use of predictors from other methods. It is unnecessary to make a function evaluation at the initial part of the new block since at all blocks (with the exception the first block) the first function evaluation is already available from the previous block.

The paper is structured as follows. Section 2 states the generalized block HBDF, its derivation from continuous approximation, and how to generate specific members of the scheme. The HBDFs are bundle as main and additional methods, a concept that is due to Brugnano and Trigiante [14]. In Section 3 we study the stability of the schemes with emphasis on a Chebyshev based member. In Section 4 a numerical algorithm for the block method is provided and implemented on selected test problems. Finally, the conclusion of the paper is discussed in Section 5. 


\section{Hybrid Backward Differentiation Type Formulas}

2.1. $k$-Step HBDF. We define a $k$-step HBDF (main method) for the numerical solution of (1) on the interval $\left[t_{n}, t_{n+k}\right]$ as

$$
\sum_{j=0}^{k} \alpha_{j} y_{n+j}+\sum_{j=1}^{k} \alpha_{v_{j}} y_{n+v_{j}}=h \beta_{k} f_{n+k}
$$

where $k \in \mathbb{Z}^{+}$is the step number, $t_{n}=t_{0}+n h, h=$ $\left(t_{N}-t_{0}\right) / N, n=0, \ldots, N, \alpha_{j}, \alpha_{v_{j}}, \beta_{k}$ are constant coefficients, and $v_{j} \in(0, k)$ are off-grid points which cannot be integers. We note that $y_{n+j} \approx y\left(t_{n+j}\right), y_{n+v_{j}} \approx y\left(t_{n+v_{j}}\right)$, and $f_{n+k}=$ $f\left(t_{n+k}, y_{n+k}\right)$. Conventionally the method (2) is applied in the predictor-corrector mode which involves the use of starting values and predictors from other methods. In this paper, a different approach that involves a block-by-block implementation is adopted, whereby additional methods are generated from a continuous scheme and combined with (2) to form a block method which is then applied to (1) without the need for starting values and predictors. In what follows, we state the block $k$-step HBDF for solving (1).

\subsection{Block HBDF. We define the block $k$-step HBDF as}

$$
\begin{aligned}
& \sum_{j=0}^{k-1} \bar{\alpha}_{j} y_{n+j}+\sum_{j=1}^{k} \bar{\alpha}_{v_{j}} y_{n+v_{j}} \\
& =h \bar{\beta}_{k} f_{n+k}+h \bar{\beta}_{i} f_{n+i}, \quad i=1, \ldots, k-1, \\
& \sum_{j=0}^{k-1} \widehat{\alpha}_{j} y_{n+j}+\sum_{j=1}^{k} \widehat{\alpha}_{v_{j}} y_{n+v_{j}} \\
& =h \widehat{\beta}_{k} f_{n+k}+h \widehat{\beta}_{v_{i}} f_{n+v_{i}}, \quad i=1, \ldots, k, \\
& \sum_{j=0}^{k} \alpha_{j} y_{n+j}+\sum_{j=1}^{k} \alpha_{v_{j}} y_{n+v_{j}}=h \beta_{k} f_{n+k},
\end{aligned}
$$

where the first two members of (3) are additional methods provided by the continuous scheme constructed in the next subsection; $\alpha_{j}, \alpha_{v_{j}}, \beta_{v_{i}}, \beta_{k}, \bar{\alpha}_{j}, \bar{\alpha}_{v_{j}}, \bar{\beta}_{v_{i}}, \bar{\beta}_{k}, \widehat{\alpha}_{j}, \widehat{\alpha}_{v_{j}}, \widehat{\beta}_{v_{i}}, \widehat{\beta}_{k}$ are constant coefficients, which are determined via the procedure given in the next subsection. The numerical solution $y_{n+j}$ is an approximation to the analytical solution $y\left(t_{n+j}\right), y_{n+v_{j}} \approx$ $y\left(t_{n+v_{j}}\right), f_{n+v_{j}}=f\left(t_{n+v_{j}}, y_{n+v_{j}}\right)$, and $f_{n+j}=f\left(t_{n+j}, y_{n+j}\right)$. In order to facilitate the analysis of (3) we express it in block form as follows:

$$
A_{1} W_{\mu}=A_{0} W_{\mu-1}+h\left[B_{1} F_{\mu}+B_{0} F_{\mu-1}\right]
$$

where

$$
\begin{gathered}
W_{\mu}=\left(y_{n+v_{1}}, \ldots, y_{n+v_{k}}, y_{n+1}, \ldots, y_{n+k}\right)^{T}, \\
W_{\mu-1}=\left(y_{n-v_{1}}, \ldots, y_{n-v_{k}}, y_{n-k+1}, \ldots, y_{n}\right)^{T}, \\
F_{\mu}=\left(f_{n+v_{1}}, \ldots, f_{n+v_{k}}, f_{n+1}, \ldots, f_{n+k}\right)^{T}, \\
F_{\mu-1}=\left(f_{n-v_{1}}, \ldots, f_{n-v_{k}}, f_{n-k+1}, \ldots, f_{n}\right)^{T},
\end{gathered}
$$

where $\mu=1, \ldots, F$ and $n=0, k, \ldots, N-k$ and $A_{i}, B_{i}$, $i=0,1$, are $2 k$ by $2 k$ matrices whose entries are given by the coefficients of (3). We note that $F$ is the number of blocks. The derivation given in the next subsection will be used to produce (4).

2.3. Derivation of Continuous $H B D F$. We provide here a generalized framework on which the schemes stated in the preceding section are produced. Let $\Upsilon_{J}$ be the linear space of polynomials of degree $\leq J$ and let $U(t) \in \Upsilon_{J}$ be a given polynomial. On the interval from $t_{n}$ to $t_{n+k}=t_{n}+k h$, the exact solution $y(t)$ for (1) is approximated by

$$
U(t)=-\sum_{j=0}^{k-1} \alpha_{j}(t) y_{n+j}-\sum_{j=1}^{k} \alpha_{v_{j}}(t) y_{n+v_{j}}+h \beta_{k}(t) f_{n+k} \text {, }
$$

where $\alpha_{v_{j}}(t)$ and $\beta_{k}(t)$ are continuous coefficients.

Let the following conditions be satisfied:

$$
\begin{aligned}
U\left(t_{n+v_{j}}\right) & =y_{n+v_{j}}, \quad j=1,2, \ldots, k, \\
U\left(t_{n+j}\right) & =y_{n+j}, \quad j=0,1,2, \ldots, k-1, \\
U^{\prime}\left(t_{k}\right) & =f_{n+k} .
\end{aligned}
$$

Further, suppose that (6) is defined by the polynomial basis functions

$$
\begin{aligned}
\alpha_{j}(t) & =\sum_{i=0}^{2 k} \alpha_{i+1, j} P_{i}(t), \quad j=\{0,1, \ldots, k-1\}, \\
\alpha_{v_{j}}(t) & =\sum_{i=0}^{2 k} \alpha_{i+1, j} P_{i}(t), \quad j=\{1, \ldots, k\}, \\
h \beta_{k}(t) & =\sum_{i=0}^{2 k} h \beta_{i+1, k} P_{i}(t),
\end{aligned}
$$

where the constants $\alpha_{i+1, j}, \alpha_{i+1, v_{j}}$, and $h \beta_{i+1, k}$ are uniquely determined.

Substituting (8) into (6) we get

$$
\begin{aligned}
U(t)= & -\sum_{j=0}^{k-1} \sum_{i=0}^{2 k} \alpha_{i+1, j} P_{i}(t) y_{n+j} \\
& -\sum_{j=1}^{k} \sum_{i=0}^{2 k} \alpha_{i+1, j} P_{i}(t) y_{n+v_{j}}+h \sum_{i=0}^{2 k} h \beta_{i+1, k} P_{i}(t) f_{n+k},
\end{aligned}
$$


which simplifies to

$$
\begin{aligned}
U(t)=\sum_{i=0}^{2 k}\{ & -\sum_{j=0}^{k-1} \alpha_{i+1, j} y_{n+j} \\
& \left.-\sum_{j=1}^{k} \alpha_{i+1, j} y_{n+v_{j}}+h \beta_{i+1, k} f_{n+k}\right\} P_{i}(t)
\end{aligned}
$$

and can be expressed as

$$
U(t)=\sum_{i=0}^{2 k} \ell_{i} P_{i}(t)
$$

where

$$
\ell_{i}=-\sum_{j=0}^{k-1} \alpha_{i+1, j} y_{n+j}-\sum_{j=1}^{k} \alpha_{i+1, j} y_{n+v_{j}}+h \beta_{i+1, k} f_{n+k} .
$$

By imposing the conditions (7) on (11), we obtain a system of $2 k+1$ equations, which can be expressed as

$$
Q L=V,
$$

where $L=\left(\ell_{0}, \ell_{1}, \ldots, \ell_{2 k}\right)^{T}, V=\left(y_{n}, y_{n+1}, \ldots, y_{n+k-1}\right.$, $\left.y_{n+v_{1}}, \ldots, y_{n+v_{k}}, f_{n+k}\right)^{T}$, and $Q$ is a matrix of dimension $(2 k+$ 1) $\times(2 k+1)$ defined as

$$
Q=\left(\begin{array}{ccc}
P_{0}\left(t_{n}\right) & \cdots & P_{2 k}\left(t_{n}\right) \\
P_{0}\left(t_{n+1}\right) & \cdots & P_{2 k}\left(t_{n+1}\right) \\
\vdots & & \vdots \\
P_{0}\left(t_{n+k-1}\right) & \cdots & P_{2 k}\left(t_{n+k-1}\right) \\
P_{0}\left(t_{n+v_{1}}\right) & \cdots & P_{2 k}\left(t_{n+v_{1}}\right) \\
\vdots & & \vdots \\
P_{0}\left(t_{n+v_{k}}\right) & \cdots & P_{2 k}\left(t_{n+v_{k}}\right) \\
P_{0}^{\prime}\left(t_{n+k}\right) & \cdots & P_{2 k}^{\prime}\left(t_{n+k}\right)
\end{array}\right)
$$

where $P_{j}(t) \in \Upsilon_{m}, j=0, \ldots, 2 k$, are basis functions. The elements of the vector $L$ are obtained using Cramer's Rule as

$$
\ell_{j}=\frac{\operatorname{det}\left(Q_{j}\right)}{\operatorname{det}(Q)}, \quad j=0,1, \ldots, 2 k,
$$

where $Q_{j}$ is obtained by replacing the $j$ th column of $Q$ by $V$. The values of $\ell_{j}$ are substituted into (11) to obtain the continuous approximation

$$
U(t)=\sum_{j=0}^{2 k} \frac{\operatorname{det}\left(Q_{j}\right)}{\operatorname{det}(Q)} P_{j}(t)
$$

which is used to generate the main and additional methods.

Remark 1. The coefficients of (6) are given by the explicit representation (16), since the two methods are equivalent. We note that the explicit representation given by (16) is only used for derivation purposes and is not recommended to be used numerically since its implementation will be expensive.
2.4. Generating Specific Members of the Method (3). We illustrate here how to generate specific members of the method (3) and in particular present the schemes for the case where the off-grid points are chosen as the zeros of Chebyshev's polynomial. If we choose $k=2$ and for notational simplicity let $\left\{v_{1}, v_{2}\right\}=\{r, s\}$, then on the interval from $t_{n}$ to $t_{n+2}=$ $t_{n}+2 h$, the approximation (6) becomes

$$
U(t)=-\sum_{j=0}^{1} \alpha_{j}(t) y_{n+j}-\sum_{j=1}^{2} \alpha_{v_{j}}(t) y_{n+v_{j}}+h \beta_{2}(t) f_{n+2},
$$

with first derivative given by

$U^{\prime}(t)$

$$
=\frac{d}{d t}\left(-\sum_{j=0}^{1} \alpha_{j}(t) y_{n+j}-\sum_{j=1}^{2} \alpha_{v_{j}}(t) y_{n+v_{j}}+h \beta_{2}(t) f_{n+2}\right),
$$

where $\alpha_{j}(t), \beta_{2}(t)$, and $\alpha_{v_{j}}(t)$ are continuous coefficients uniquely determined as

$$
\begin{aligned}
& \alpha_{0}=\sigma_{1}\left(-12 z+17 r z-5 r^{2} z+17 s z-24 r s z\right. \\
& +7 r^{2} s z-5 s^{2} z+7 r s^{2} z-2 r^{2} s^{2} z-16 z^{2} \\
& +11 r z^{2}-2 r^{2} z^{2}+11 s z^{2}+r s z^{2}-2 r^{2} s z^{2} \\
& -2 s^{2} z^{2}-2 r s^{2} z^{2}+r^{2} s^{2} z^{2}+4 z^{3}-9 r z^{3} \\
& +3 r^{2} z^{3}-9 s z^{3}+6 r s z^{3}-r^{2} s z^{3}+3 s^{2} z^{3} \\
& \left.-r s^{2} z^{3}+8 z^{4}-3 r z^{4}-3 s z^{4}+r s z^{4}\right), \\
& \alpha_{1}=\sigma_{2}\left(20-28 r+8 r^{2}-28 s+39 r s-11 r^{2} s\right. \\
& +8 s^{2}-11 r s^{2}+3 r^{2} s^{2}+48 z-48 r z+12 r^{2} z \\
& -48 s z+40 r s z-9 r^{2} s z+12 s^{2} z-9 r s^{2} z \\
& +2 r^{2} s^{2} z+24 z^{2}-8 r z^{2}-8 s z^{2}-6 r s z^{2} \\
& +3 r^{2} s z^{2}+3 r s^{2} z^{2}-r^{2} s^{2} z^{2}-16 z^{3}+16 r z^{3} \\
& -4 r^{2} z^{3}+16 s z^{3}-8 r s z^{3}+r^{2} s z^{3} \\
& \left.-4 s^{2} z^{3}+r s^{2} z^{3}-12 z^{4}+4 r z^{4}+4 s z^{4}-r s z^{4}\right) \\
& \alpha_{r}=\sigma_{3}\left(12 z-17 s z+5 s^{2} z+16 z^{2}-11 s z^{2}\right. \\
& \left.+2 s^{2} z^{2}-4 z^{3}+9 s z^{3}-3 s^{2} z^{3}-8 z^{4}+3 s z^{4}\right), \\
& \alpha_{s}=\sigma_{4}\left(-12 z+17 r z-5 r^{2} z-16 z^{2}+11 r z^{2}\right. \\
& \left.-2 r^{2} z^{2}+4 z^{3}-9 r z^{3}+3 r^{2} z^{3}+8 z^{4}-3 r z^{4}\right), \\
& \beta_{2}=\rho_{1}^{-1}\left(z-r z-s z+r s z+3 z^{2}-2 r z^{2}\right. \\
& \left.-2 s z^{2}+r s z^{2}+3 z^{3}-r z^{3}-s z^{3}+z^{4}\right),
\end{aligned}
$$


and where $\rho_{1}=20-8 r-8 s+3 r s, \sigma_{1}=\left(r s \rho_{1}\right)^{-1}, \sigma_{2}=$ $\left[(r-1)(s-1) \rho_{1}\right]^{-1}, \sigma_{3}=\left[(r-1) r(r-s) \rho_{1}\right]^{-1}$, and $\sigma_{4}=$ $\left[(r-s)(s-1) s \rho_{1}\right]^{-1}$. We also introduce the scale variable $z$ for convenience only by letting $z=\left(t-t_{n+1}\right) / h$.

Suppose that the off-grid points are specified by the zeros of the second degree Chebyshev polynomial of the first kind given by $r=(1 / 2)(2-\sqrt{2})$ and $s=(1 / 2)(2+\sqrt{2})$; then $(18)$ yields three additional methods when evaluated at $t=\left\{t_{n}+\right.$ $\left.h, t_{n}+h r, t_{n}+h s\right\}$ which are combined with the main method obtained by evaluating (17) at $t=t_{n}+2 h$ to get a Chebyshev based method of the form (3) given as

$$
\begin{aligned}
& 11 h f_{n+1}+h f_{n+2} \\
& =-(4+9 \sqrt{2}) y_{n+r}-(4-9 \sqrt{2}) y_{n+s}+6 y_{n} \\
& +2 y_{n+1} \\
& 2(-2+\sqrt{2}) h f_{n+2}+22 h f_{n+r} \\
& =-2(12+5 \sqrt{2}) y_{n}+(8-26 \sqrt{2}) y_{n+1} \\
& -(-24+5 \sqrt{2}) y_{n+r}+(-8+11 \sqrt{2}) y_{n+s} \text {, } \\
& 2(2+\sqrt{2}) h f_{n+2}-22 h f_{n+s} \\
& =-2(-12+5 \sqrt{2}) y_{n} \\
& +(8+11 \sqrt{2}) y_{n+r}-(24+5 \sqrt{2}) y_{n+s} \\
& +(8+26 \sqrt{2}) y_{n+1} \text {, } \\
& 2 h f_{n+2}=-4(2+\sqrt{2}) y_{n+s}+y_{n}+4 y_{n+1}+11 y_{n+2} \\
& +4(-2+\sqrt{2}) y_{n+r} \text {, }
\end{aligned}
$$

whose stability properties are discussed in the next section.

\section{Stability of Block HBDF}

In this section, we discuss the stability of the block HBDF (4) with emphasis on the Chebyshev based method (20). We pause to remark that the method (20) is a specific member of the class of methods (3) and therefore a similar analysis can be generated for other members.

Suppose the local truncation error of the block method (4) is defined as

$$
\mathrm{LTE}=\mathbf{Z}_{\mu}-\left(A_{1}^{-1} A_{0} \mathbf{Z}_{\mu-1}+h A_{1}^{-1}\left[B_{1} \overline{\mathbf{F}}_{\mu}+B_{0} \overline{\mathbf{F}}_{\mu-1}\right]\right),
$$

where

$$
\begin{gathered}
\mathbf{Z}_{\mu}=\left(y\left(t_{n+v_{1}}\right), \ldots, y\left(t_{n+v_{k}}\right), y\left(t_{n+1}\right), \ldots, y\left(t_{n+k}\right)\right)^{T}, \\
\overline{\mathbf{F}}_{\mu}=\left(f\left(t_{n+v_{1}}, y\left(t_{n+v_{1}}\right)\right), \ldots, f\left(t_{n+v_{k}}, y\left(t_{n+v_{k}}\right)\right),\right. \\
\left.\quad f\left(t_{n+1}, y\left(t_{n+1}\right)\right), \ldots, f\left(t_{n+k}, y\left(t_{n+k}\right)\right)\right)^{T}, \\
\mathbf{Z}_{\mu-1}=\left(y\left(t_{n-v_{1}}\right), \ldots, y\left(t_{n-v_{k}}\right), y\left(t_{n-k+1}\right), \ldots, y\left(t_{n}\right)\right)^{T}, \\
\overline{\mathbf{F}}_{\mu-1}=\left(f\left(t_{n-v_{1}}, y\left(t_{n-v_{1}}\right)\right), \ldots, f\left(t_{n-v_{k}}, y\left(t_{n-v_{k}}\right)\right),\right. \\
\left.f\left(t_{n-k+1}, y\left(t_{n-k+1}\right)\right), \ldots, f\left(t_{n}, y\left(t_{n}\right)\right)\right)^{T} .
\end{gathered}
$$

We recall here that the block method (4) is characterized by the matrices $A_{i}, B_{i}, i=0,1$, whose entries are given by the coefficients of (3).

Definition 2. The block method (4) has order of consistency $p \geq 1$.

Thus, we expand the terms in (21) as a Taylor series about the point $t_{n}$ and also assume that the constant coefficients $C_{q}=0$ for $q=0,1, \ldots, p$, leads to the local truncation error satisfying the maximum norm $\|\mathrm{LTE}\|=C_{p+1} h^{p+1}+O\left(h^{p+2}\right)$.

Theorem 3. The block method (4) is zero-stable.

Proof. Zero-stability is concerned with the stability of a difference system in the limit as $h$ tends to zero. Thus, as $h \rightarrow 0$ and noting that the first $2 k-1$ columns of $A_{0}$ are all zeros with nonzero elements occurring only in the last column, the normalized first characteristic polynomial $\rho(\chi)$ of the method (4) is given by

$$
\rho(\chi)=\operatorname{det}\left(\chi I-A_{1}^{-1} A_{0}\right)=\Gamma_{1} \chi^{2 K-1}(\chi+1) .
$$

$\Gamma_{1}$ is a constant and $I$ is the identity matrix. Following Fatunla [15], the block method (4) is zero-stable, since from (23), $\rho(\chi)=0$ satisfy $\left|\chi_{j}\right| \leq 1, j=1, \ldots, 2 k$, and for those roots with $\left|\chi_{j}\right|=1$, the multiplicity does not exceed 1 .

In particular, the Chebyshev based method (20) is zerostable as a consequence of Theorem 3 , since our analysis shows that its associated characteristic polynomial $\rho(\chi)$ is given by $\rho(\chi)=-(48 / 11) \chi^{3}(\chi+1)$. Thus, the root condition is satisfied, since for $\rho(\chi)=0, \chi_{j}=\{0,0,0,-1\}, j=1, \ldots, 4$, and for those roots with $\left|\chi_{j}\right|=1$, the multiplicity does not exceed 1 .

Lemma 4. The block method (4) is convergent.

Proof. Following Henrici [16] that convergence $=$ consistency + zero-stability, the proof is a consequence of Definition 2 and Theorem 3. 
TABle 1: A comparison of the Errs of methods for Example 1.

\begin{tabular}{lllcccccc}
\hline Steps & HBDF & Rate & BVM & Rate & Ad-IVM & Rate & BDF & $8.7 \times 10^{-2}$ \\
\hline 20 & $1.5 \times 10^{-2}$ & & $1.2 \times 10^{-1}$ & & $4.0 \times 10^{-1}$ & & Rate \\
40 & $2.3 \times 10^{-3}$ & 2.7 & $1.8 \times 10^{-2}$ & 2.8 & $2.4 \times 10^{-2}$ & 4.0 & $6.8 \times 10^{-2}$ \\
80 & $9.9 \times 10^{-5}$ & 4.2 & $1.6 \times 10^{-3}$ & 3.5 & $1.6 \times 10^{-3}$ & 3.9 & $1.1 \times 10^{-2}$ \\
160 & $5.9 \times 10^{-6}$ & 4.1 & $1.4 \times 10^{-4}$ & 3.5 & $1.2 \times 10^{-4}$ & 3.7 & $8.0 \times 10^{-4}$ & 3.6 \\
320 & $3.3 \times 10^{-7}$ & 4.2 & $1.1 \times 10^{-5}$ & 3.7 & $1.1 \times 10^{-5}$ & 3.5 & $6.8 \times 10^{-5}$ & 3.6 \\
640 & $1.9 \times 10^{-8}$ & 4.1 & $7.4 \times 10^{-7}$ & 3.9 & $7.5 \times 10^{-7}$ & 3.8 & $5.3 \times 10^{-6}$ \\
\hline
\end{tabular}

TABLE 2: A comparison of errors of methods at $t=5$ for Example 2.

\begin{tabular}{lccc}
\hline$h$ & HBDF & Gauss-2s & EF-Gauss-2s \\
\hline 0.1 & $5.4907 \times 10^{-11}$ & $5.1207 \times 10^{-6}$ & $8.3555 \times 10^{-7}$ \\
0.01 & $6.0665 \times 10^{-15}$ & $6.4579 \times 10^{-12}$ & $4.2408 \times 10^{-12}$ \\
\hline
\end{tabular}

The stability of the method is studied next. The application of (4) to the test equation $y^{\prime}=\lambda y$ gives the following discrete problem:

$$
W_{\mu}=M(q) W_{\mu-1}, \quad q=\lambda h
$$

where $M(q)=\left(A_{1}-q B_{1}\right)^{-1}\left(A_{0}+q B_{0}\right)$ is the stability matrix.

Definition 5. The block method (4) is said to be (i) $A$-stable if for all $q \in \mathbb{C}^{-}, M(q)$ has a dominant eigenvalue $q_{\max }$ such that $\left|q_{\max }\right| \leq 1$; moreover, since $q_{\max }$ is a rational function, the real part of the zeros of $q_{\max }$ must be negative, while the real part of the poles of $q_{\max }$ must be positive; (ii) $A_{0}$-stable if for all $q \in \Re \subset \mathbb{C}^{-}, M(q)$ has a dominant eigenvalue $q_{\max }$ such that $\left|q_{\max }\right| \leq 1$; (iii) $L_{0}$-stable if it is $A_{0}$-stable and $q_{\max } \rightarrow 0$ as $q \rightarrow-\infty$; and (iv) $L$-stable if it is $A$-stable and $q_{\max } \rightarrow 0$ as $q \rightarrow-\infty$.

Definition 6. Let the stability region of (4) be $\Omega$; then the method (4) is said to be $A(\alpha)$-stable, $\alpha \in(0, \pi / 2)$, if $\Omega \supset$ $\Omega_{\alpha}=\{q:|\arg (-q)|<\alpha, q \neq 0\}$.

Theorem 7. The block HBDF (4) is $L_{0}$-stable and $A(\alpha)$-stable.

Proof. The eigenvalues of the stability matrix $M(q)$ in (24) are rational functions of $q$. It can be easily shown that the dominant eigenvalue $q_{\max }$ has the form $q_{\max }=\varphi(q) / \psi(q)$, where the degree of the polynomial $\psi(q)$ is greater than that of $\varphi(q)$ and $|\varphi(q)| \leq|\psi(q)|$. Applying Definitions 5 and 6 with $\alpha=90^{\circ}$ completes the proof.

Theorem 8. The Chebyshev based method (20) is $L_{0}$-stable and $A(\alpha)$-stable.

Proof. The dominant eigenvalue $q_{\max }$ for the Chebyshev based method is given by

$$
q_{\max }=-\frac{48+36 q+10 q^{2}+q^{3}}{48-60 q+34 q^{2}-11 q^{3}+2 q^{4}} .
$$

The proof follows from Theorem 7.

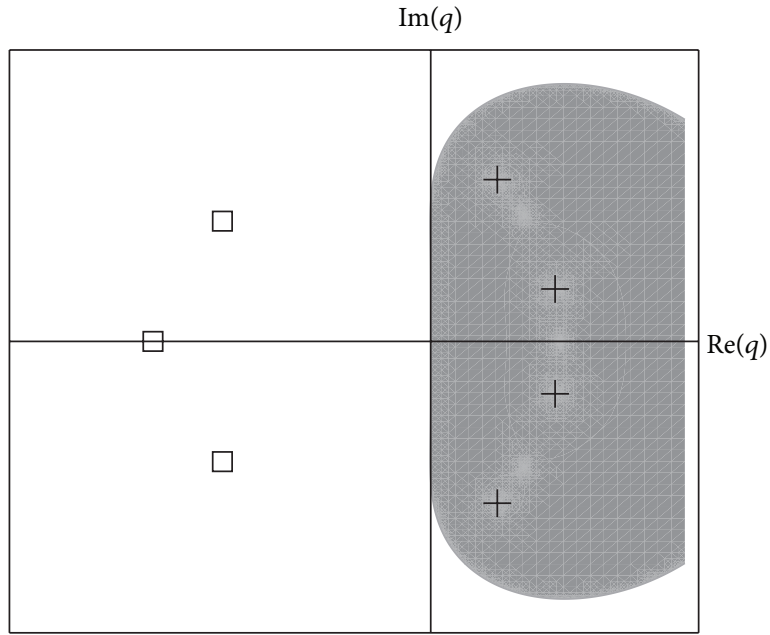

FIGURE 1: The region of absolute stability of the Chebyshev based HBDF of order 4 is to the left of the dividing line and is symmetric about the real axis; the square and plus symbols to the left and right of the imaginary axis represent the zeros and poles of $q_{\max }$, respectively.

Moreover, the stability region for the Chebyshev based method is given in Figure 1 showing the zeros and poles of the dominant eigenvalue $q_{\max }$.

\section{Numerical Application}

4.1. Block Algorithm. Given the interval $\left[t_{0}, t_{N}\right]$ where $t_{n}=$ $t_{0}+n h$ for $n=0,1, \ldots, N$, the method (3) is implemented in block form (4) as follows.

Step 1. Let $k=2$; the method (3) reduces to the Chebyshev based method (20), and for $\mu=1$, and $n=0$, the values of $W_{1}=\left(u_{r}, u_{1}, u_{s}, u_{2}\right)^{T}$ ( $T$ is the transpose) are simultaneously obtained over the subinterval $\left[t_{0}, t_{2}\right]$ since $W_{0}$ is known from the system of ODEs.

Step 2. For $\mu=2, n=2$, the values of $W_{2}=\left(u_{r+2}, u_{3}\right.$, $\left.u_{s+2}, u_{4}\right)^{T}$ are simultaneously obtained over the subinterval $\left[t_{2}, t_{4}\right]$ since the values of $W_{1}$ are known from the previous block.

Step 3. For $\mu \geq 3$, the process is continued to generate the vectors for the approximate solutions of the ODE on subintervals $\left[t_{4}, t_{6}\right], \ldots,\left[t_{N-2}, t_{N}\right]$. 
TABLE 3: Absolute errors, $\left(\operatorname{Err}\left(y_{i}\right)=\left|y(t)-y_{i}\right|\right), i=1,2,3$, for Example 3 .

\begin{tabular}{|c|c|c|c|c|c|c|c|}
\hline \multirow{2}{*}{ Method } & \multirow{2}{*}{$h$} & \multicolumn{3}{|c|}{$t=2$} & \multicolumn{3}{|c|}{$t=48$} \\
\hline & & $\operatorname{Err}\left(y_{1}\right)$ & $\operatorname{Err}\left(y_{2}\right)$ & $\operatorname{Err}\left(y_{3}\right)$ & $\operatorname{Err}\left(y_{1}\right)$ & $\operatorname{Err}\left(y_{2}\right)$ & $\operatorname{Err}\left(y_{3}\right)$ \\
\hline \multirow{5}{*}{$\mathrm{HBDF}$} & 1 & $5.24 \times 10^{-10}$ & $4.47 \times 10^{-9}$ & $4.99 \times 10^{-9}$ & $1.31 \times 10^{-14}$ & $3.36 \times 10^{-9}$ & $3.36 \times 10^{-9}$ \\
\hline & $1 / 2$ & $2.82 \times 10^{-13}$ & $2.31 \times 10^{-9}$ & $2.31 \times 10^{-9}$ & $6.60 \times 10^{-15}$ & $1.69 \times 10^{-9}$ & $1.69 \times 10^{-9}$ \\
\hline & $1 / 4$ & $6.02 \times 10^{-15}$ & $1.15 \times 10^{-9}$ & $1.15 \times 10^{-9}$ & $3.30 \times 10^{-15}$ & $8.45 \times 10^{-10}$ & $8.46 \times 10^{-10}$ \\
\hline & $1 / 8$ & $2.99 \times 10^{-15}$ & $5.73 \times 10^{-10}$ & $5.73 \times 10^{-10}$ & $1.64 \times 10^{-15}$ & $4.19 \times 10^{-10}$ & $4.22 \times 10^{-10}$ \\
\hline & $1 / 16$ & $1.48 \times 10^{-15}$ & $2.82 \times 10^{-10}$ & $2.83 \times 10^{-10}$ & $8.07 \times 10^{-16}$ & $2.06 \times 10^{-10}$ & $2.10 \times 10^{-10}$ \\
\hline
\end{tabular}

TABLE 4: A comparison of errors of methods for Example 4 at $t=1$.

\begin{tabular}{ccccccc}
\hline$\Delta x$ & $\Delta t$ & $\kappa$ & $\begin{array}{c}\text { Crank- } \\
\text { Nicolson }\end{array}$ & Cash (ab) & Cash (abc) & $(\mathrm{HBDF})$ \\
\hline 0.1 & 0.1 & 1 & $3.0 \times 10^{-5}$ & $1.5 \times 10^{-5}$ & $4.5 \times 10^{-6}$ & $1.43 \times 10^{-6}$ \\
0.1 & 0.1 & 5 & $2.0 \times 10^{-4}$ & $3.0 \times 10^{-8}$ & $2.0 \times 10^{-10}$ & $3.66 \times 10^{-15}$ \\
\hline
\end{tabular}

4.2. Numerical Examples for Stiff ODEs. We will implement the block algorithm described above using the Chebyshev based method (20) for three different stiff systems given in the literature. The first example shows the performance of the block method on a linear stiff system. The next two examples are chosen to demonstrate the performance of the block method on a $2 \times 2$ and a $3 \times 3$ nonlinear stiff system.

Example 1. Consider the following linear system on the range $0 \leq t \leq 1: y_{1}^{\prime}=-21 y_{1}+19 y_{2}-20 y_{3}, y_{2}^{\prime}=19 y_{1}-21 y_{2}+20 y_{3}$, and $y_{3}^{\prime}=40 y_{1}-40 y_{2}-40 y_{3}$ with initial conditions $y_{1}(0)=1$, $y_{2}(0)=0$, and $y_{3}(0)=-1$, respectively. The exact solution of the system is given by $y_{1}(t)=(1 / 2)\left(e^{-2 t}+e^{-40 t}(\cos (40 t)+\right.$ $\sin (40 t))), y_{2}(t)=(1 / 2)\left(e^{-2 t}-e^{-40 t}(\cos (40 t)+\sin (40 t))\right)$, and $y_{3}(t)=(1 / 2)\left(2 e^{-40 t}(\sin (40 t)-\cos (40 t))\right)$.

This problem has also been solved by Amodio and Mazzia [17] using the boundary value methods (BVMs), implicit Adams methods (Ad-IVMs), and backward differentiation formulas (BDFs). The results for their order 4 methods are reproduced in Table 1 and compared with the results given by the HBDF which is also of order 4. It is seen from Table 1 that the HBDF performs better than those in [17]. In all cases, the calculated rate of convergence of each method is consistent with the theoretical order $(p=4)$ behavior of each method. In particular, it is obvious from Table 1 that HBDF exhibits an order 4 behavior, since on halving the step size, the error is reduced by a factor of about 18, which can be expressed approximately as $2^{4}=16$. Thus, for this example, HBDF is superior in terms of accuracy. We note that the maximum relative errors for the first component (Errs) displayed in Table 1 are computed as $\max (|y-y(t)| /(1+|y(t)|))$.

Example 2. We consider the following IVP which was solved by Vaquero and Vigo-Aguiar, [18]: $y_{1}^{\prime}=-1002 y_{1}+1000 y_{2}^{2}$ and $y_{2}^{\prime}=y_{1}-y_{2}\left(1+y_{2}\right)$ with initial conditions $y_{1}(0)=1$ and $y_{2}(0)=1$, respectively. The exact solution is $y_{1}(t)=e^{-2 t}$; $y_{2}(t)=e^{-t}$.
The results for this problem obtained by Vaquero and Vigo-Aguiar [18] using an exponentially fitted Gauss (EFGauss-2s) and Gauss-2s methods of order 4 are reproduced in Table 2 and compared with the results given by HBDF which is also of order 4 . It is seen from Table 2 that the HBDF performs better than those in [18].

Example 3. We consider the given nonlinear system on the range $0 \leq t \leq 48$ :

$$
\begin{gathered}
y_{1}^{\prime}=-0.013 y_{2}-1000 y_{1} y_{2}-2500 y_{1} y_{3}, \quad y_{1}(0)=0, \\
y_{2}^{\prime}=-0.013 y_{2}-1000 y_{1} y_{2}, \quad y_{2}(0)=1, \\
y_{3}^{\prime}=-2500 y_{1} y_{3}, \quad y_{3}(0)=1 .
\end{gathered}
$$

True solution is

$$
\begin{aligned}
& y_{i}(2)=\{-3.616933169289 \times 10^{-6}, \\
&0.9815029948230,1.018493388244\}, \\
& y_{i}(48)=\left\{-1.945338956808 \times 10^{-6},\right. \\
&0.6110474831446,1.388950571516\}, \\
& i=1,2,3
\end{aligned}
$$

(see Jeltsch [19]).

This example was chosen to show the performance of the HBDF on a $3 \times 3$ nonlinear stiff system. In Table 3 , the accuracy of the HBDF is measured by the maximum computing absolute errors at $t=\{2,48\}$.

4.3. Numerical Examples for Parabolic PDEs. In this section we test the block algorithm with Chebyshev based scheme (20) on large systems derived from parabolic pdes. Consider the homogeneous partial differential equation

$$
\frac{\partial u}{\partial t}=\frac{\partial^{2} u}{\partial x^{2}}, \quad(x, t) \in[0,1] \times\left[0<t \leq T_{N}\right],
$$

subject to the initial/boundary conditions $u(x, 0)=f(x), x \in$ $[0,1], u(0, t)=u(1, t)=0$, and $t \geq 0$.

Suppose that (28) is discretized using the method of lines in such as way that the resulting system of ordinary differential equations is stable (see Lambert [20], Ramos and 
TABLE 5: A comparison of errors of methods for Example 5 at $t=1$ and $\bar{\omega}=1, \Delta x=0.1, \Delta t=0.1$.

\begin{tabular}{lcccc}
\hline $\bar{\omega}$ & $(\mathrm{HBDF})$ & Crank-Nicolson & Cash (ab) & Cash (abc) \\
\hline 1 & $2.87 \times 10^{-6}$ & $6.20 \times 10^{-5}$ & $3.7 \times 10^{-5}$ & $1.5 \times 10^{-5}$ \\
2 & $1.43 \times 10^{-6}$ & $3.83 \times 10^{-5}$ & $1.8 \times 10^{-5}$ & $7.4 \times 10^{-6}$ \\
3 & $1.43 \times 10^{-6}$ & $9.30 \times 10^{-3}$ & $1.9 \times 10^{-5}$ & $7.4 \times 10^{-6}$ \\
5 & $1.43 \times 10^{-6}$ & $1.80 \times 10^{-1}$ & $1.8 \times 10^{-5}$ & $7.4 \times 10^{-6}$ \\
10 & $1.43 \times 10^{-6}$ & $6.10 \times 10^{-1}$ & $1.8 \times 10^{-5}$ & $7.4 \times 10^{-6}$ \\
\hline
\end{tabular}

Vigo-Aguiar [21], and Cash [2]). Let $\mathbf{u}(t) \in \mathfrak{R}^{m}$ be the exact solution of the resulting semidiscrete problem

$$
\frac{d \mathbf{u}(t)}{d t}=A \mathbf{u}(t), \quad U(0)=\phi
$$

where $\mathbf{u}(t)=\left[u_{1}(t), u_{2}(t), \ldots, u_{M}(t)\right]^{T}, u_{m}(t) \approx u\left(x_{m}, t\right), \phi=$ $\left[\phi\left(x_{1}\right), \phi\left(x_{2}\right), \ldots, \phi\left(x_{M}\right)\right]^{T}$, and

$$
A=\frac{1}{(\Delta x)^{2}}\left[\begin{array}{ccccc}
-2 & 1 & & & \\
1 & -2 & 1 & & 0 \\
& \ddots & \ddots & \ddots & \\
0 & & 1 & -2 & 1 \\
& & & 1 & -2
\end{array}\right]
$$

The problem (29) is now a system of ordinary differential equations on which the block algorithm can be applied. We remark that if $\Delta x$ is small, the system (29) is stiff.

Example 4. Consider the given PDE (see Cash [2])

$$
\begin{gathered}
\frac{\partial u}{\partial t}=\kappa \frac{\partial^{2} u}{\partial x^{2}} \\
u(0, t)=u(1, t)=0, \quad u(x, 0)=\sin \pi x .
\end{gathered}
$$

The exact solution $u(x, t)=e^{-\pi^{2} \kappa t} \sin \pi x$.

The output in Table 4 compares the performance of the block method with those given in Cash [2]. It is clear from the table that the block method is the more accurate.

Example 5. Consider the given stiff parabolic equation (see Cash [2])

$$
\begin{gathered}
\frac{\partial u}{\partial t}=\kappa \frac{\partial^{2} u}{\partial x^{2}}, \\
u(0, t)=u(1, t), \quad u(x, 0)=\sin \pi x+\sin \bar{\omega} \pi x, \quad \bar{\omega} \gg 1 .
\end{gathered}
$$

The exact solution $u(x, t)=e^{-\pi^{2} \kappa t} \sin \pi x+e^{-\bar{\omega}^{2} \pi^{2} \kappa t} \sin \bar{\omega} \pi x$.

Cash [2] notes that as $\bar{\omega}$ increases, equations of the type given in Example 5 exhibit characteristics similar to model stiff equations. Hence, methods such as the Crank-Nicolson method which are not $L_{0}$-stable are expected to perform poorly. The block HBDF is $L$-stable and hence performs well on this problem. Table 5 displays the results for $\kappa=1$ and a range of values for $\bar{\omega}$.

\section{Conclusion}

In this paper, we have proposed a generalized high order block hybrid $k$-step HBDF for the numerical solution of stiff systems, which includes large systems arising from semidiscretized parabolic PDEs. A specific scheme based on the zeros of the second degree Chebyshev polynomial of the first kind was generated and its stability established. Applications of the block algorithm to stiff ODEs and PDEs demonstrate the accuracy of the method. The block algorithm is facilitated by the availability of the continuous method which has the ability to generate additional methods, which are combined and applied in block form. We remark that the block-by-block approach has stability and accuracy advantages over the stepby-step methods which are generally applied in a predictorcorrector mode. Particularly, the block-by-block approach gives smaller global errors at the end of the interval compared to those produced by the step-by-step methods due to the fact that the accumulation of error at each step is inherent in the step-by-step methods. Our future research will be focused on applying continuous methods to delay and differential algebraic equations.

\section{Conflict of Interests}

The authors declare that there is no conflict of interests regarding the publication of this paper.

\section{References}

[1] L. W. Jackson and S. K. Kenue, "A fourth order exponentially fitted method," SIAM Journal on Numerical Analysis, vol. 11, pp. 965-978, 1974.

[2] J. R. Cash, "Two new finite difference schemes for parabolic equations," SIAM Journal on Numerical Analysis, vol. 21, no. 3, pp. 433-446, 1984.

[3] W. H. Enright, "Continuous numerical methods for ODEs with defect control," Journal of Computational and Applied Mathematics, vol. 125, no. 1-2, pp. 159-170, 2000.

[4] P. Onumanyi, U. W. Sirisena, and S. N. Jator, "Continuous finite difference approximations for solving differential equations," International Journal of Computer Mathematics, vol. 72, no. 1, pp. 15-27, 1999.

[5] P. Onumanyi, D. O. Awoyemi, S. N. Jator, and U. W. Sirisena, "New linear multistep methods with continuous coefficients for first order initial value problems," Journal of the Nigerian Mathematical Society, vol. 13, pp. 37-51, 1994. 
[6] O. A. Akinfenwa, S. N. Jator, and N. M. Yao, "Continuous block backward differentiation formula for solving stiff ordinary differential equations," Computers \& Mathematics with Applications, vol. 65, no. 7, pp. 996-1005, 2013.

[7] S. N. Jator, "On the hybrid method with three off-step points for initial value problems," International Journal of Mathematical Education in Science and Technology, vol. 41, no. 1, pp. 110-118, 2010.

[8] L. F. Shampine and H. A. Watts, "Block implicit one-step methods," Mathematics of Computation, vol. 23, pp. 731-740, 1969.

[9] P. Chartier, " $L$-stable parallel one-block methods for ordinary differential equations," SIAM Journal on Numerical Analysis, vol. 31, no. 2, pp. 552-571, 1994.

[10] J. D. Rosser, "A Runge-kutta for all seasons," SIAM Review, vol. 9, pp. 417-452, 1967.

[11] M. T. Chu and H. Hamilton, "Parallel solution of ODE's by multi-block methods," SIAM Journal on Scientific and Statistical Computing, vol. 8, no. 3, pp. 342-353, 1987.

[12] S. N. Jator, S. Swindell, and R. French, "Trigonometrically fitted block Numerov type method for $y^{\prime \prime}=f\left(x, y, y^{\prime}\right)$," Numerical Algorithms, vol. 62, no. 1, pp. 13-26, 2013.

[13] S. N. Jator, "Leaping type algorithms for parabolic partial differential equations," in International Conference on Scientific Computing, Abuja, Nigeria, August 2011.

[14] L. Brugnano and D. Trigiante, Solving Differential Problems by Multistep Initial and Boundary Value Methods, Gordon and Breach Science Publishers, Amsterdam, Netherlands, 1998.

[15] S. O. Fatunla, "Block methods for second order IVPs," International Journal of Computer Mathematics, vol. 41, pp. 55-63, 1991.

[16] P. Henrici, Discrete Variable Methods in ODEs, John Wiley \& Sons, 1962.

[17] P. Amodio and F. Mazzia, "Boundary value methods based on Adams-type methods," Applied Numerical Mathematics, vol. 18, no. 1-3, pp. 23-35, 1995.

[18] J. M. Vaquero and J. Vigo-Aguiar, "Exponential fitted RungeKutta methods of collocation type based on Gauss, Radau, and Labatto traditional methods," in Proceedings of the International Conference on Computational and Mathematical Methods in Science and Engineering (CMMSE '07), pp. 289-303, 2007.

[19] R. Jeltsch, "Multistep methods using higher derivatives and damping at infinity," Mathematics of Computation, vol. 31, no. 137, pp. 124-138, 1977.

[20] J. D. Lambert, Numerical Methods for Ordinary Differential Systems, John Wiley \& Sons, New York, NY, USA, 1991.

[21] H. Ramos and J. Vigo-Aguiar, "A fourth-order runge-kutta method based on BDF-type chebyshev approximations," Journal of Computational and Applied Mathematics, vol. 204, no. 1, pp. 124-136, 2007. 


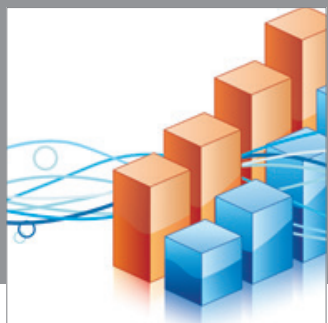

Advances in

Operations Research

mansans

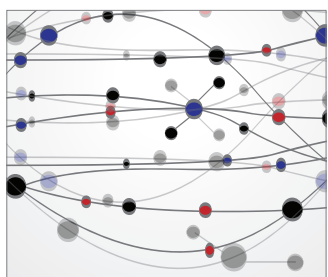

The Scientific World Journal
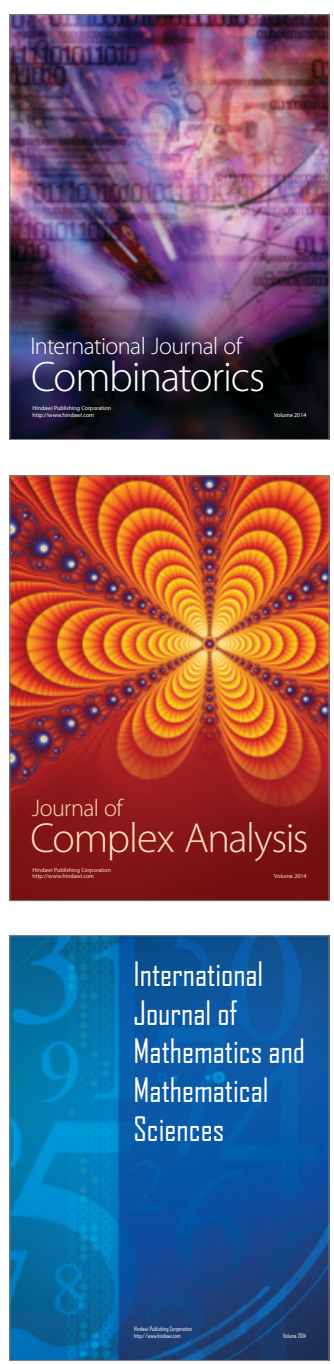
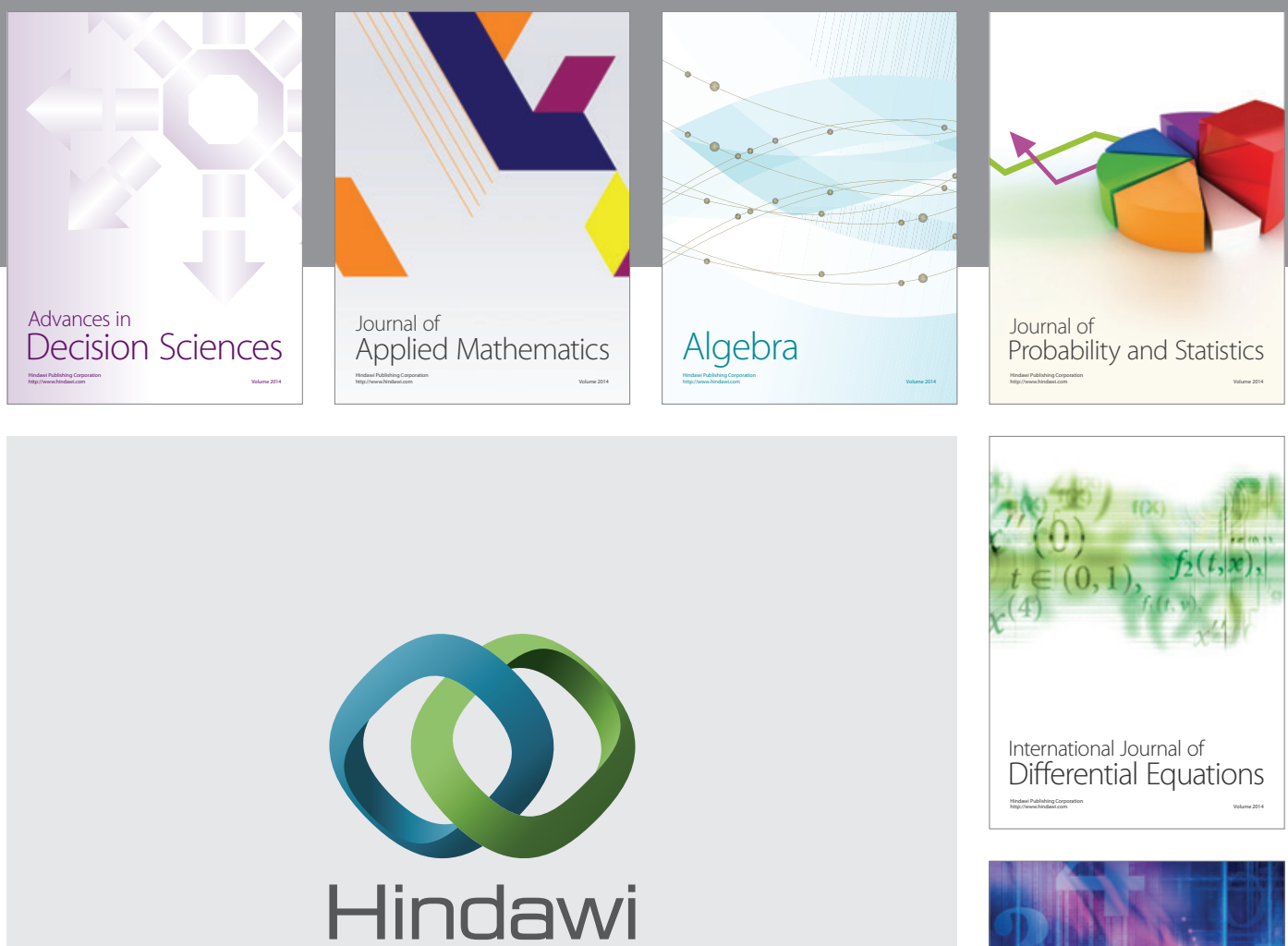

Submit your manuscripts at http://www.hindawi.com
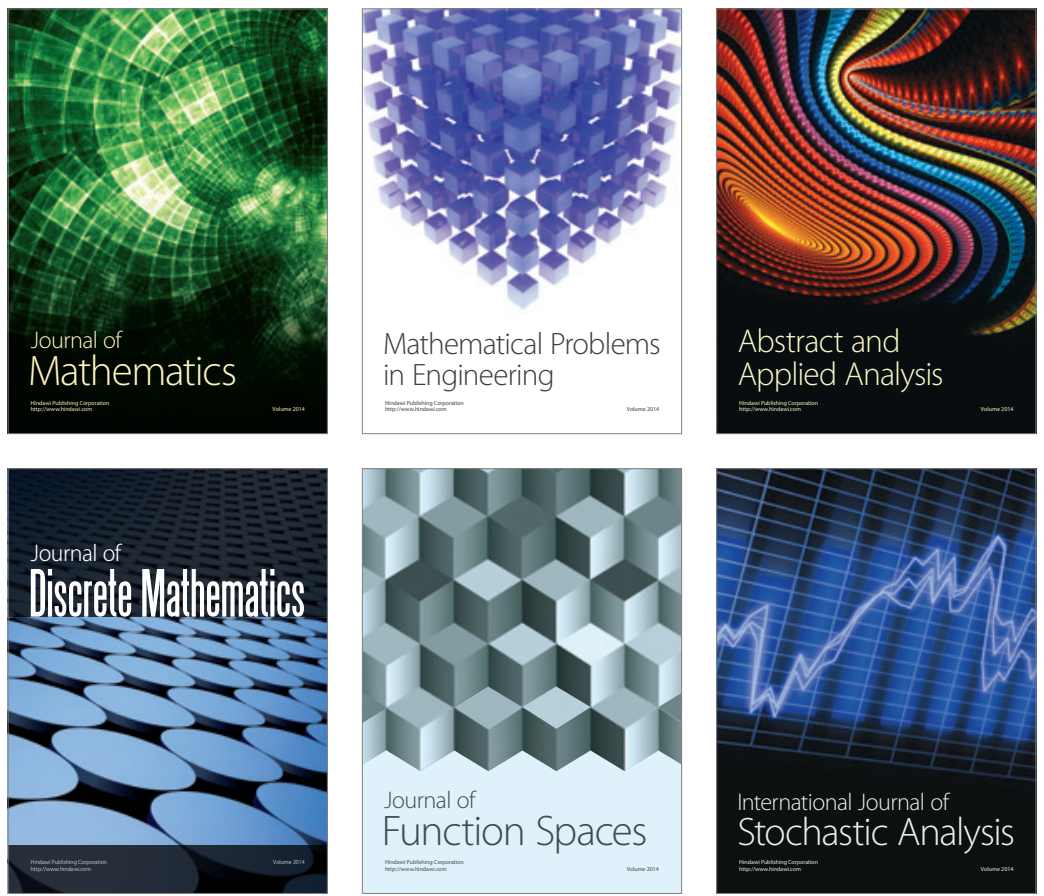

Journal of

Function Spaces

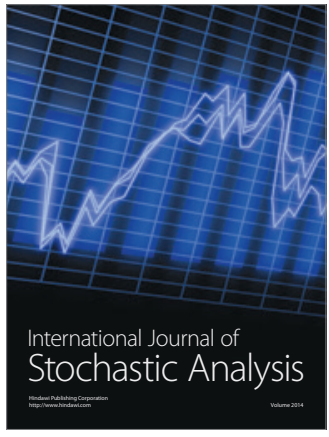

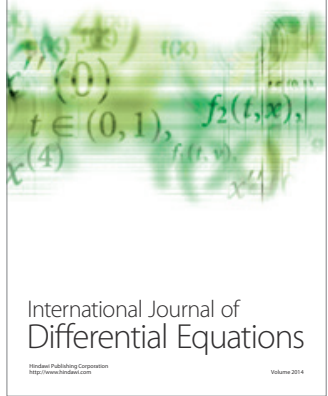
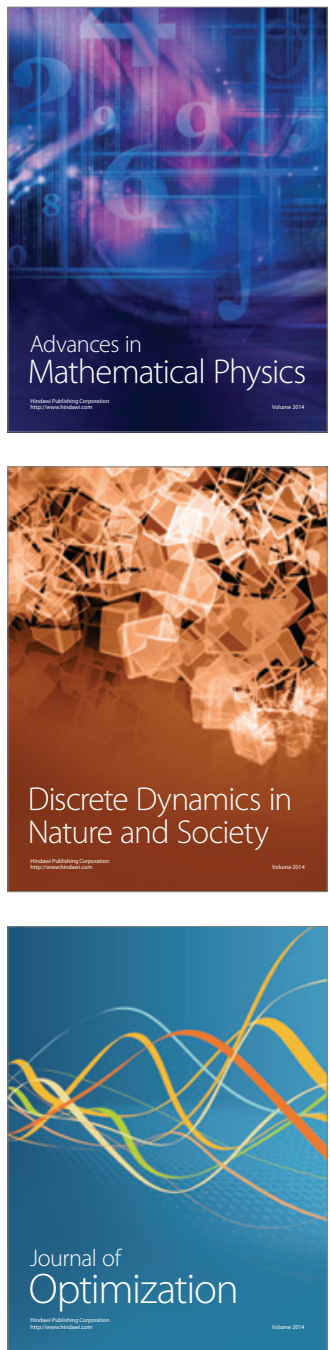